\title{
The Dynamic Modelling and Simulation of Spatial Multibody Systems with Prismatic Joint Based on Vector Bond Graph
}

\author{
Zhongshuang Wang ${ }^{1, a}$, Yangyang Tao ${ }^{2, b}$, Shang Liu ${ }^{3, c}$ \\ School of Mechatronics Engineering \\ Qiqihar University \\ Qiqihar, China \\ E-mail: wzhsh1962@163.com, bcrazy_for_you_5@163.com, cshang liu 2009@126.com
}

\begin{abstract}
For the modelling and simulation of complex spatial multibody systems, the vector bond graph method is proposed. By the kinematic constraint condation, spatial prismatic joint can be modeled by vector bond graph. For the algebraic difficulties brought by differential causality in system automatic modeling and simulation, the effective decoupling method is proposed and the differential causalities in system vector bond graph model can be eliminated. In the case of considering EJS, the unified formulae of system state space equations and constraint forces at joints are derived, which are easily derived on a computer and very suitable for spatial multibody systems. As a result, the unified modelling and simulation for complex spatial multibody systems are realized, its validity is illustrated by a practical example.
\end{abstract}

Keywords-Vector bond graph; Modelling and simulation; Spatial multibody system; Prismatic joint; Causality

\section{INTRODUCTION}

System modeling and simulation are very important to the control and dynamic design of modern mechanical systems. For complex spatial multibody systems, e.g. the spatial multibody systems with prismatic joint , deriving system state space equations and constraint force equations at joints is a very tedious and erroprone task. Although different procedures have been proposed to increase the reliability and efficiency of this process ${ }^{[1,2]}$, most of them can not be used to deal with systems that simultaneously include various physical domains in a unified manner.

Bond graph technique ${ }^{[3]}$ was chosen because it is a computer oriented method which can describe all type of physical systems, thus allowing a single model to represent the dynamic interactions of the spatial multibody system with electrical, hydraulic, pneumatic, and other components. Compared with scalar bond graph ${ }^{[3]}$, vector bond graph is more suitable for modelling spatial multibody systems because of its more concise representation manner ${ }^{[4,5]}$. But for spatial multibody systems, the kinematic and geometric constraints between bodies result in differential causality loop, and the nonlinear velocity relationship between the mass center and an arbitrary point on a body leads to the nonlinear junction strcture. Current vector bond graph procedures ${ }^{[4]}$ were found to be very difficult algebraically in derivation of system state space equations automatically on a computer.To solve above problems, a more efficient and practical modelling and simulation procedure for spatial multibody systems with prismatic joint based on vector bond graph $^{[4]}$ is proposed here.

\section{VECTOR BOND GRAPH MODEL OF SPATIAL MULTIBODY SYSYEM WITH PRISMATIC JOINT}

In any spatial multi-body system, the joints impose kinematic constraints on the rigid body elements. A general rigid body moving in space can be modelled by vector bond graph $^{[4]}$. The diagram of spatial prismatic joint is shown in Figure.1, this constraint limits the relative translation of the two bodies $\mathrm{B}_{\alpha}$ and $\mathrm{B}_{\beta}$ along two directions, and limits the relative rotation of the two bodies $\mathrm{B}_{\alpha}$ and $\mathrm{B}_{\beta}$ along three directions. Joint point $\mathrm{P}$ and $\mathrm{Q}$ are fixed on rigid body $\mathrm{B}_{\alpha}$ and $\mathrm{B}_{\beta}$ respectively, vector $h_{\alpha}$ is used to describe the relative motion of the two rigid bodies, $h_{\alpha}=r_{\alpha}^{P}-r_{\beta}^{Q}$. Where $r_{\alpha}^{P}$ and $r_{\beta}^{Q}$ represent the position vector of joint point $\mathrm{P}$ and $\mathrm{Q}$ in global coordinates respectively, $r_{\alpha}^{P}=\left[\begin{array}{lll}x_{\alpha}^{P} & y_{\alpha}^{P} & z_{\alpha}^{P}\end{array}\right]^{T}, r_{\beta}^{Q}=\left[\begin{array}{ccc}x_{\beta}^{Q} & y_{\beta}^{Q} & z_{\beta}^{Q}\end{array}\right]^{T} \cdot d_{\beta}^{1}$ and $d_{\beta}^{2}$ are two unit vectors fixed on rigid body $\mathrm{B}_{\beta}$, which are all orthogonal to axis, and orthogonal to each other. $d_{\alpha}$ is the unit vector fixed on rigid body $\mathrm{B}_{\alpha}$ along axis, $d_{\alpha}^{1}$ is another unit vector fixed on rigid body $\mathrm{B}_{\alpha}$ and paralell to $d_{\beta}^{1} . \quad d_{\alpha}^{\prime}, \quad d_{\alpha}^{\prime 1}, \quad d_{\beta}^{\prime 1}$ and $d_{\beta}^{\prime 2}$ are the corresponding

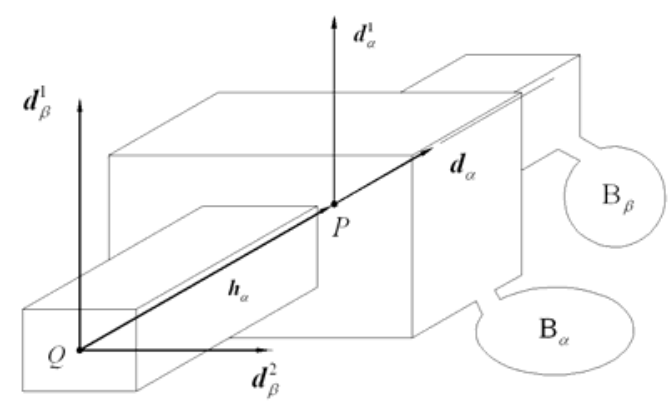

Figure.1 A sketch of prismatic joint 
vectors in body frame. From the Kinematic constraint condition of prismatic joint ${ }^{[1]}$, the position constraint equations can be written as

$$
\begin{aligned}
& \underline{\Phi}^{\left(d_{2}\right)}\left(h_{\alpha}, d_{\beta}^{1}, d_{\beta}^{2}\right)=\left[\begin{array}{l}
d_{\beta}^{1 T} h_{\alpha} \\
d_{\beta}^{2 T} h_{\alpha}
\end{array}\right]=\left[\begin{array}{l}
d_{\beta}^{\prime 1 T} A^{\beta T}\left(r_{\beta}^{Q}-r_{\alpha}^{P}\right) \\
d_{\beta}^{\prime 2 T} A^{\beta T}\left(r_{\beta}^{Q}-r_{\alpha}^{P}\right)
\end{array}\right]=0 \\
& \underline{\Phi}^{\left(r_{3}\right)}\left(d_{\alpha}, d_{\alpha}^{1}, d_{\beta}^{1}, d_{\beta}^{2}\right)=\left[\begin{array}{l}
d_{\beta}^{1 T} d_{\alpha} \\
d_{\beta}^{2 T} d_{\alpha} \\
d_{\beta}^{2 T} d_{\alpha}^{1}
\end{array}\right]=\left[\begin{array}{c}
d_{\beta}^{\prime 1 T} A^{\beta T} A^{\alpha} d_{\alpha}^{\prime} \\
d_{\beta}^{\prime 2 T} A^{\beta T} A^{\alpha} d_{\alpha}^{\prime} \\
d_{\beta}^{\prime 2 T} A^{\beta T} A^{\alpha} d_{\alpha}^{\prime 1}
\end{array}\right]=0
\end{aligned}
$$

where $A^{\beta}$ is the direction cosin matrix of body $\mathrm{B}_{\beta}$.

The corresponding velocity(or angular velocity) constraint equations can be written as

$$
\begin{gathered}
\underline{\Phi}^{\left(r_{3}\right)}\left(d_{\alpha}, d_{\alpha}^{1}, d_{\beta}^{1}, d_{\beta}^{2}\right)=\left[\omega_{\alpha}-\omega_{\beta}\right]=0 \\
\dot{\Phi}^{\left(d_{2}\right)}\left(h_{\alpha}, d_{\beta}^{1}, d_{\beta}^{2}\right)=\left[\begin{array}{c}
d_{\beta}^{1 T} \dot{h}_{\alpha}+h_{\alpha}^{T} \dot{d}_{\beta}^{1} \\
d_{\beta}^{2 T} \dot{h}_{\alpha}+h_{\alpha}^{T} \dot{d}_{\beta}^{2}
\end{array}\right] \\
=\left[\begin{array}{c}
d_{\beta}^{\prime 1 T} A^{\beta T}\left(\dot{r}_{\alpha}^{P}-\dot{r}_{\beta}^{Q}\right)-h_{\alpha}^{T} \tilde{d}_{\beta}^{1} \omega_{\beta} \\
d_{\beta}^{\prime 2 T} A^{\beta T}\left(\dot{r}_{\alpha}^{P}-\dot{r}_{\beta}^{Q}\right)-h_{\alpha}^{T} \tilde{d}_{\beta}^{2} \omega_{\beta}
\end{array}\right]=0
\end{gathered}
$$

where $\omega_{\alpha}$ and $\omega_{\beta}$ represent the angular velocity vector of the rigid body determined in global coordinates.

$$
\tilde{d}_{\beta}^{i}=\left[\begin{array}{ccc}
0 & -d_{\beta z}^{i} & d_{\beta y}^{i} \\
d_{\beta z}^{i} & 0 & -d_{\beta x}^{i} \\
-d_{\beta y}^{i} & d_{\beta x}^{i} & 0
\end{array}\right] \quad(i=1,2)
$$

The velocity(or angular velocity) constraint equations shown as Eqs.(3) and Eqs.(4) can be presented by vector bond model shown as Figure.2.

The vector bond graph for the rigid body undergoing spatial motion can be coupled to one another satisfying the kinematic constraints ${ }^{[1-2]}$ at the interfaces to get the over system model. But the kinematic constraints result in differential causality. In the derivation of system state space equations, the current vector bond graph procedures ${ }^{[4]}$ were found to be very difficult algebraically. To eliminate the differential causality, the constraint force vectors at joints can be considered as unknown effort source vectors and

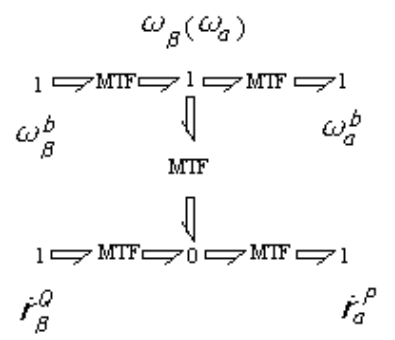

Figure.2 Vector bond graph model of prismatic join added to the corresponding 0 -junctions of the system vector bond graph model.

\section{The Unified Formulae OF System State Space EQUATIONS AND CONSTRAINT FORCES}

The basic field and junction structure of system bond graph is imposed is shown in Figure. $3^{[3]}$, where Eulerjunction structure $^{[4]}$ (EJS) is added. $X_{i_{l}}$ represents energy vector variable of independent storage energy field corresponding to independent motion, $X_{i_{2}}$ represents energy vector variable of independent storage energy field corresponding to dependent motion, $Z_{i_{1}}$ and $Z_{i_{2}}$ are the corresponding coenergy vector variables. $D_{\text {in }}$ and $D_{\text {out }}$ represent input and output vector variables in resistive field, $U$ and $V$ represent input and output vector variables of source field respectively, $U=\left[\begin{array}{ll}U_{1} & U_{2}\end{array}\right]^{T}, V=\left[\begin{array}{ll}V_{1} & V_{2}\end{array}\right]^{T}$. Where $U_{1}$ is known source vector, and $U_{2}$ represents the constraint force vector of joint. $E_{\text {in }}$ and $E_{\text {out }}$ are the input and output vector variables in Euler-junction structure (EJS).

For independent energy storage field, we have

$$
\begin{aligned}
& Z_{i_{1}}=F_{i_{1}} X_{i_{1}} \\
& Z_{i_{2}}=F_{i_{2}} X_{i_{2}}
\end{aligned}
$$

where $F_{i_{1}}$ and $F_{i_{2}}$ are the $m_{1} \times m_{1}$ and $m_{2} \times m_{2}$ matrices respectively.

For resistive field, we have

$$
D_{\text {out }}=R D_{\text {in }}
$$

where $R$ is $L \times L$ matrix.

For Euler-junction structure (EJS), we have

$$
E_{\text {out }}=R_{E} E_{\text {in }}
$$

where $R_{E}$ is $L_{E} \times L_{E}$ matrix $^{[6]}$.

The corresponding junction structure equations can be written as

$\dot{X}_{i_{1}}=J_{i_{1} i_{1}} Z_{i_{1}}+J_{i_{1} i_{2}} Z_{i_{2}}+J_{i_{1} L} D_{\text {out }}+J_{i_{1} u_{1}} U_{1}+J_{i_{1} u_{2}} U_{2}+J_{i_{1} E} E_{\text {out }}$

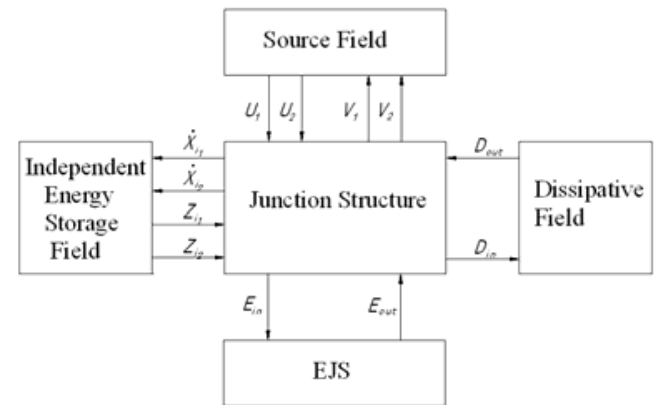


Figure.3 Basic field and junction structure of system

$$
\begin{aligned}
& \dot{X}_{i_{2}}=J_{i_{i_{1}} I_{1}} Z_{i_{l}}+J_{i_{2} i_{2}} Z_{i_{2}}+J_{i_{2} L} D_{\text {out }}+J_{i_{2} u_{l}} U_{1}+J_{i_{2} u_{2}} U_{2}+J_{i_{2} E} E_{\text {out }} \\
& D_{\text {in }}=J_{L i_{l}} Z_{i_{l}}+J_{L i_{2}} Z_{i_{2}}+J_{L L} D_{\text {out }}+J_{L u_{l}} U_{1}+J_{L u_{2}} U_{2}+J_{L E} E_{\text {out }} \\
& E_{\text {in }}=J_{E i_{l}} Z_{i_{I}}+J_{E i_{2}} Z_{i_{2}}+J_{E L} D_{\text {out }}+J_{E u_{l}} U_{1}+J_{E u_{2}} U_{2}+J_{E E} E_{\text {out }}
\end{aligned}
$$

From the flow summation of 0 -junctions corresponding to $m_{2}$ constraint force vectors in system vector bond graph model, we have

$$
0=J_{C_{i_{1}}} Z_{i_{1}}+J_{C_{i_{2}} Z_{i_{2}}}+J_{C L} D_{\text {out }}+J_{C u_{1}} U_{1}+J_{C E} E_{\text {out }}
$$

By the algebraic manipulation from (5) (13), the system state space equations can be written as

If $\operatorname{det}\left(T_{L E}\right) \neq 0$

$$
\begin{aligned}
& \dot{X}_{i_{I}}=T_{i_{I} i_{I}} X_{i_{I}}+T_{i_{1} i_{2}} X_{i_{2}}+T_{i_{1} u_{I}} U_{1}+T_{i_{1} u_{2}} U_{2} \\
& \dot{X}_{i_{2}}=T_{i_{2} i_{1}} X_{i_{I}}+T_{i_{2} i_{2}} X_{i_{2}}+T_{i_{2} u_{I}} U_{1}+T_{i_{2} u_{2}} U_{2} \\
& U_{2}=-T_{L E}^{-1}\left(T_{u_{2} i_{1}} X_{i_{1}}+T_{u_{2} i_{2}} X_{i_{2}}+T_{u_{2} u_{I}} U_{1}\right)
\end{aligned}
$$

where

$$
\begin{aligned}
& A_{1}=\left[I_{2}-J_{E E} R_{E}-J_{E L} R\left(I_{1}-J_{L L} R\right)^{-1} J_{L E} R_{E}\right]^{-1} \\
& A_{2}=J_{E i_{1}} F_{i_{1}}+J_{E L} R\left(I_{1}-J_{L L} R\right)^{-1} J_{L i_{1}} F_{i_{1}} \\
& A_{3}=J_{E i_{2}} F_{i_{2}}+J_{E L} R\left(I_{1}-J_{L L} R\right)^{-1} J_{L i_{2}} F_{i_{2}} \\
& A_{4}=J_{E u_{1}}+J_{E L} R\left(I_{1}-J_{L L} R\right)^{-1} J_{L u_{t}} \\
& A_{5}=J_{E u_{2}}+J_{E L} R\left(I_{1}-J_{L L} R\right)^{-1} J_{L u_{2}} \\
& B_{1}=\left(I_{1}-J_{L L} R\right)^{-1}\left(J_{L i_{l}} F_{i_{I}}+J_{L E} R_{E} A_{1} A_{2}\right) \\
& B_{2}=\left(I_{1}-J_{L L} R\right)^{-1}\left(J_{L i_{2}} F_{i_{2}}+J_{L E} R_{E} A_{1} A_{3}\right) \\
& B_{3}=\left(I_{1}-J_{L L} R\right)^{-1}\left(J_{L u_{I}} F_{i_{I}}+J_{L E} R_{E} A_{1} A_{2}\right) \\
& B_{4}=\left(I_{1}-J_{L L} R\right)^{-1}\left(J_{L u_{2}} F_{i_{1}}+J_{L E} R_{E} A_{1} A_{5}\right) \\
& T_{i_{1} i_{l}}=J_{i_{1} i_{l}} F_{i_{l}}+J_{i_{L} L} R B_{1}+J_{i_{l} E} R_{E} A_{1} A_{2} \\
& T_{i_{1} i_{2}}=J_{i_{l} i_{2}} F_{i_{2}}+J_{i_{l} L} R B_{2}+J_{i_{1} E} R_{E} A_{1} A_{3} \\
& T_{i_{1} u_{1}}=J_{i_{l} u_{1}}+J_{i_{l} L} R B_{3}+J_{i_{L} E} R_{E} A_{1} A_{4} \\
& T_{i_{1} u_{2}}=J_{i_{1} u_{2}}+J_{i_{1} L} R B_{4}+J_{i_{1} E} R_{E} A_{1} A_{5} \\
& T_{i_{2} i_{1}}=J_{i_{2} i_{1}} F_{i_{1}}+J_{i_{2} L} R B_{1}+J_{i_{2} E} R_{E} A_{1} A_{2} \\
& T_{i_{2} i_{2}}=J_{i_{2} i_{2}} F_{i_{2}}+J_{i_{2} L} R B_{2}+J_{i_{2} E} R_{E} A_{1} A_{3} \\
& T_{i_{2} u_{1}}=J_{i_{2} u_{1}}+J_{i_{2} L} R B_{3}+J_{i_{2} E} R_{E} A_{1} A_{4} \\
& T_{i_{2} u_{2}}=J_{i_{2} u_{2}}+J_{i_{2} L} R B_{4}+J_{i_{2} E} R_{E} A_{1} A_{5} \\
& T_{L E}=J_{C L} R B_{4}+J_{C E} R_{E} A_{1} A_{5} \\
& T_{u_{2} i_{1}}=J_{C i_{1}} F_{i_{1}}+J_{C L} R B_{1}+J_{C E} R_{E} A_{1} A_{2} \\
& T_{u_{2} i_{2}}=J_{C_{2}} F_{i_{2}}+J_{C L} R B_{2}+J_{C E} R_{E} A_{1} A_{3}
\end{aligned}
$$

$$
T_{u_{2} u_{I}}=J_{C u_{I}}+J_{C L} R B_{3}+J_{C E} R_{E} A_{1} A_{4}
$$

If $J_{C L}=0, J_{C E}=0$

$$
\begin{aligned}
& \dot{X}_{i_{I}}=T_{i_{I} i_{I}} X_{i_{I}}+T_{i_{1} i_{2}} X_{i_{2}}+T_{i_{1} u_{I}} U_{1}+T_{i_{1} u_{2}} U_{2} \\
& \dot{X}_{i_{2}}=T_{i_{2} i_{I}} X_{i_{I}}+T_{i_{2} i_{2}} X_{i_{2}}+T_{i_{2} u_{I}} U_{1}+T_{i_{2} u_{2}} U_{2} \\
& U_{2}=-H_{4}^{-1}\left(H_{1} X_{i_{I}}+H_{2} X_{i_{2}}+H_{3} U_{1}+J_{C_{I}} \dot{U}_{1}\right)
\end{aligned}
$$

where

$$
\begin{aligned}
& H_{1}=\dot{J}_{C_{i_{I}}} F_{i_{1}}+J_{C_{i_{1}}} F_{i_{1}} T_{i_{1} i_{2}}+J_{C_{i_{2}}} F_{i_{2}} T_{i_{2} i_{I}}, \\
& H_{2}=\dot{J}_{C_{i_{2}}} F_{i_{2}}+J_{C_{i_{1}}} F_{i_{1}} T_{i_{1} i_{2}}+J_{C_{i_{2}}} F_{i_{2}} T_{i_{2} i_{2}} \\
& H_{3}=\dot{J}_{C u_{I}}+J_{C i_{I}} F_{i_{I}} T_{i_{1} u_{1}}+J_{C_{i}} F_{i_{2}} T_{i_{2} u_{I}} \text {, } \\
& H_{4}=J_{C_{i_{1}}} F_{i_{1}} T_{i_{1} u_{2}}+J_{C_{i}} F_{i_{2}} T_{i_{2} u_{2}}
\end{aligned}
$$

For the system state space equations shown as (14) or (15), giving the initial value of state variable vector $X_{i_{1}}$, $X_{i_{2}}$, the constraint force vector $U_{2}$ can be obtained from (14c) or (15c). Thus (14a) and (14b) or (15a) and (15b) is a set of first order differential equations, many numerical solving algorithm that are available can be used. The corrected adaptive step size Runge-Kutta method based on MATLAB program ${ }^{[7]}$ is emplored here.

\section{EXAMPLE SYSTEM}

Figure.4 shows a spatial multibody system. The components for this example are three rigid bodies, two revolute joints $\mathrm{J}_{1}, \mathrm{~J}_{2}$ and a prismatic joint $\mathrm{J}_{3}$. These components are parameterized with the following data: $\mathrm{m}_{1}=\mathrm{m}_{2}=1 \mathrm{Kg}$ are the mass of the rigid body $\mathrm{m}_{1}$ and $\mathrm{m}_{2}$, $\mathrm{I}_{\mathrm{xx}}=\mathrm{I}_{\mathrm{yy}}=\mathrm{I}_{\mathrm{zz}}=4.167 \mathrm{e}-4 \mathrm{Kgm}^{2}$ are the principal moment of inertia of $\mathrm{m}_{1}$ and $\mathrm{m}_{2} \cdot \mathrm{F}_{1}=\mathrm{F}_{2}=-9.8 \mathrm{~N}$ are the weight of $\mathrm{m}_{1}$ and $\mathrm{m}_{2} \cdot \mathrm{M}=0.1 \mathrm{~N} \cdot \mathrm{m}$ is a moment imposed to $J_{1}, K=500 N / m$ is spring stfffness, $a=0.3 m$, $b=0.1 \mathrm{~m}, \mathrm{c}=0.15 \mathrm{~m}$ are the distances shown in Figure. 4 .

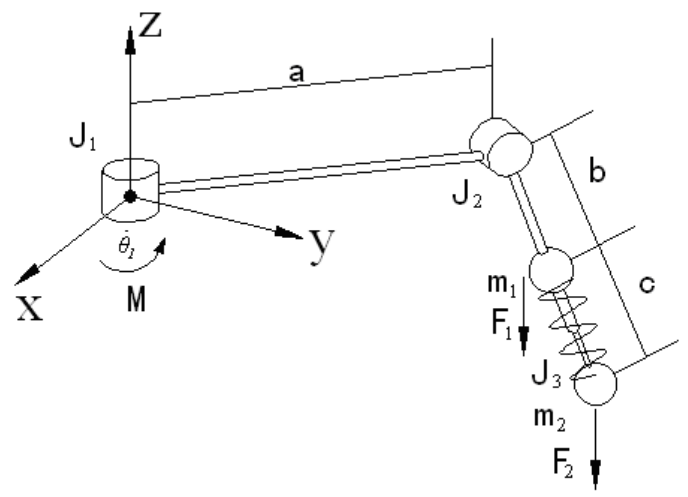

Figure.4 Spatial multibody system 
For revolute joint, three translations and two rotational degrees of freedom are constrainted, leaving only one Rotation degree of freedom free. From this constraint condition, its vector bond graph can be obtained. By assembling the vector bond graph of a single space moving rigid body, the revolute joints, and the prismatc joint, the overal system vector bond graph model can be obtained and shown as Figure.5. The constraint force vectors of joints can be considered as unknown source vectors, such as $S e_{2}, S e_{5}$, $S e_{7}, S e_{10}$ in Figure.5, and added to the corresponding 0junctions to eliminate differential causality. As a result, all differential causalities in this system vector bond graph can be eliminated, thus the procedure presented here can be used.

In Figure.5,

$$
J_{t_{z}}=\left[\mathrm{I}_{\mathrm{zZ}}\right] \quad \text {, }
$$

$\mathcal{J}_{2}^{b}=\operatorname{diag}\left[\mathrm{I}_{\mathrm{XX}} \quad \mathrm{I}_{\mathrm{YY}} \mathrm{I}_{\mathrm{ZZ}}\right]$,

$J_{3}^{b}=\operatorname{diag}\left[\mathrm{I}_{\mathrm{XX}} \quad \mathrm{I}_{\mathrm{YY}} \quad \mathrm{I}_{\mathrm{ZZ}}\right] \cdot \dot{r}_{c_{2}}, \dot{r}_{c_{3}}$ are the mass center

velocity vector of body $\mathrm{m}_{1}$ and $\mathrm{m}_{2}$ in global coordinates, $\omega_{2}^{b}, \omega_{3}^{b}$ are the angular velocity vector of body $m_{1}$ and $\mathrm{m}_{2}$ in body frame respectively.

Inputting the initial values of state variable vector, the physical parameters of the mechanism, and the coefficent matrices of (5) (13) into the program associated with the procedure presented here based on MATLAB ${ }^{[7]}$, the system responses and the resultant constraint forces at joints are obtained and shown in Figure.6 Figure.9. These results are good agreement with that obtained by the procedure in [5].

\section{CONCLUSIONS}

A general procedure was presented for using vector bond graph to model spatial multibody systems with prismatc joint. Compared with standard scalar bond graph model, the procedure presented here is more concise. Because nonlinear junction structure and differential causality exit in shch complex systems, current vector bond graph methods are found to be very difficult algebraically in the derivation of system state space equations. The constraint force vectors of joints can be considered as unknown source vectors, and added to the corresponding 0 -junctions to eliminate differential causality. In the case of considering EJS, the unified formulae of system state space equations and constraint forces at joints are derived, which are easily derived on a computer and very suitable for spatial multibody systems. These lead to a more efficient and practical automated procedure for modelling and simulation of complex spatial multibody systems over a multi-enegy domains in a unified manner.

\section{ACKNOWLEDGMENT}

This work was financially supported by National Natural Science Foundation of China (Grant No. 51175272).

\section{REFERENCES}

[1] J. Z. Hong. Computational Dynamics of Multibody Systems. Beijing: The Advanced Education Press, 2003

[2] L. P. Chen,, Y. Q. Zhang, W. Q. Ren, et al. .Mechanical System Dynamcs and ADAMS Application Course. Beijing:Tsinghua University Press, 2005

[3] D. C. Karnopp, D. L. Margolis, R. C. Rosenberg, System Dynamics: Modeling and Simulation of Mechatronic Systems, 4th ed., New York: John Wiley, 2006

[4] S Behzadipour, A Khajepour. Causality in Vector Bond Graph and Its Application to Modelling of Multi-body Dynamic Systems.Simulation Modelling Practice and Theory, 2006: 14: 279 295

[5] G Fillippni, D Delarmelina, J Pagano, et al. Dynamics of Multibody Systems With Bond Graphs. Mecảnia Computacional., 2007: XX VI:2943 2958

[6] P. Breedveld. Stability of Rigid Rotation from a Bond Graph Pespective. Simulation Modelling Practice and Theory[J]. 2009, (17):92 106

[7] J. F. Jiang, L. J. Hu, J. T. Tang. Numerical Analysis and MATLAB Experiment. Beijing: Science Press, 2004

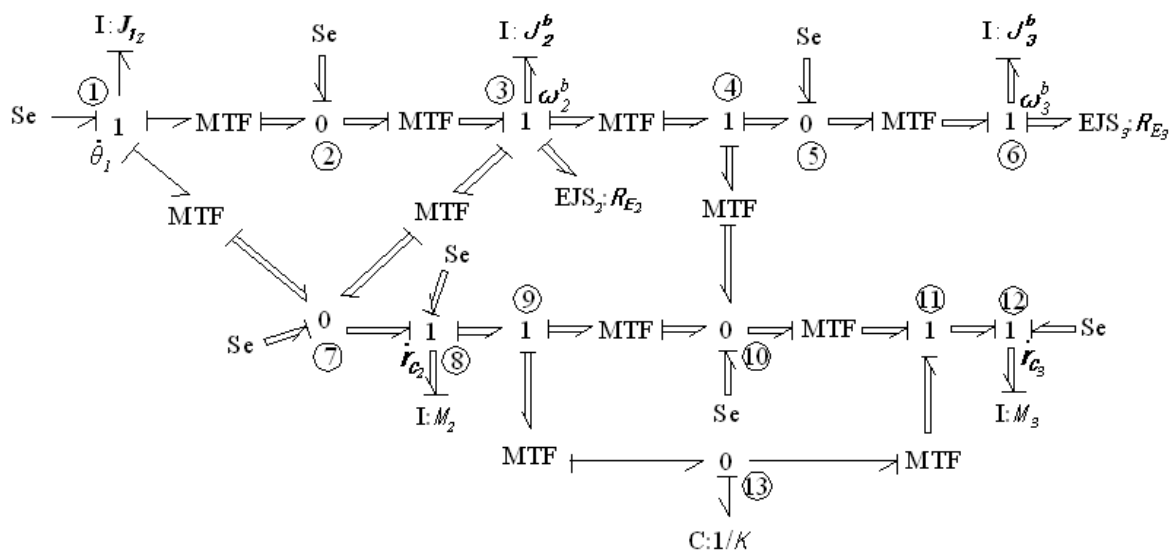


Figure.5 Vector bond graph model of system

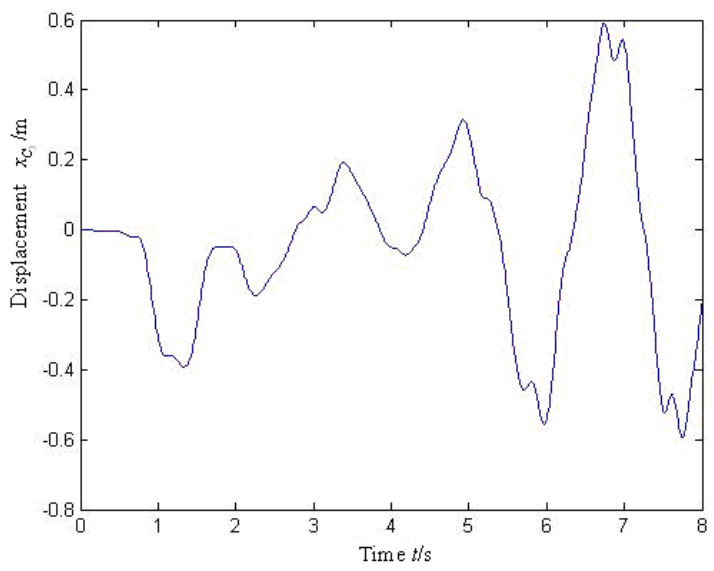

Figure.6 Displacement of $\mathrm{m}_{2}$ in global $\mathrm{X}$-axis

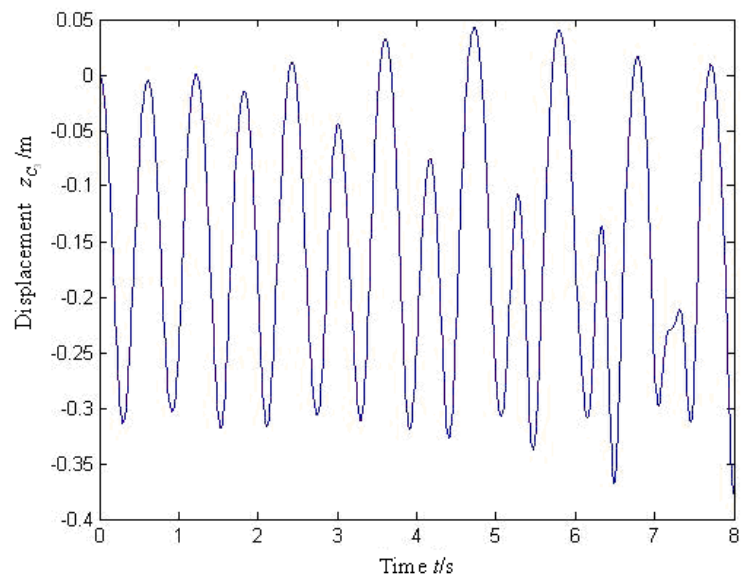

Figure.8 Displacement of $\mathrm{m}_{2}$ in global $\mathrm{Z}$-axis

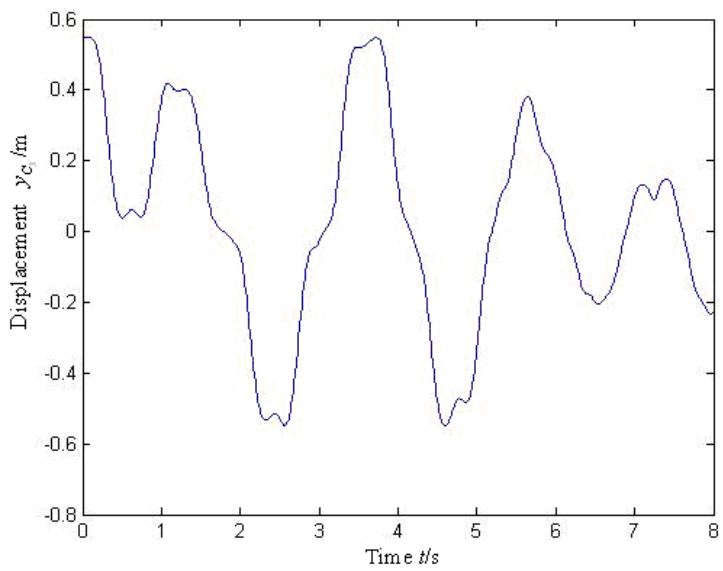

Figure.7 Displacement of $\mathrm{m}_{2}$ in global $\mathrm{Y}$-axis

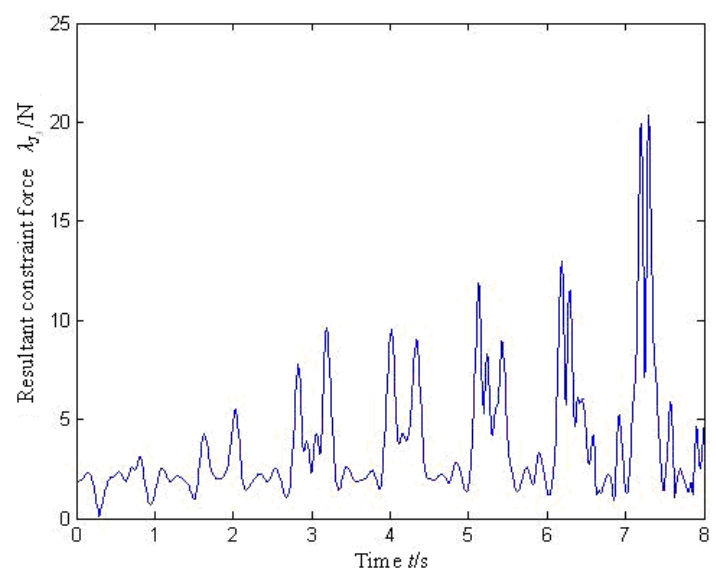

Figure.9 Resultant constraint force at joint $\mathrm{J}_{3}$ 